\title{
Survey on Finding of Admissible Information and Influential Users
}

\author{
Sharik Ahmad ${ }^{1}$, Dr. M. Mazhar Afzal ${ }^{2}$, Dr. Umesh Chandra ${ }^{3}$ \\ ${ }^{1}$ Department of Computer Science, Glocal University, Saharanpur \\ 2 Department of Computer Science, Glocal University, Saharanpur \\ ${ }^{3}$ Computer Science, Banda University of Agriculture and Technology, Banda
}

\begin{abstract}
Influential users perform a crucial aspect in online media since users serve to have a strike to each other. In this paper, Influential's originate relevant information which satisfies the information needs of a compelling dissemination information and further how specific behaviour information may grant the recognization about influence communications in an online media. Understanding information dissemination processes that existing in online media, is an elementary mark approaching to the construction of powerful information dissemination system, support system, and growing marketing skills in the digital environment. Moreover, there are couple of major approaches of information dissemination are influence and relevance. Strength of popularise information in a social media is the influence.
\end{abstract}

Keywords - Social Network Analysis, Influence / Influential Users, Relevance, Information dissemination, Profile-Rank, Page-Rank.

\section{Introduction}

In recent past, social networks have grow into prominent manner. The tremendous demand of social media sites like Twitter, Facebook, Hi5, and YouTube has lifted the Internet towards social networks (i.e., connections) and their representation. Social networking is a connection of peoples and their relationships. At social media, peoples can interact to each other in which they generate huge amounts of data. The data spawned over social media can be classified in two main mining i.e. information mining and structure mining. The data includes in information mining are metadata and XML data (i.e. text, images, audio, video etc.). Network analysis of the node and graph of connections are including in structure mining.

Social network analysis (SNA) is the survey of online media to finding and extracting of useful different patterns from semi-structured datasets which may be provincial and universal, to determine the influential users and to investigate the gesture in the network which undergo in structure mining category. So social network analysis associate a systematic analysis of social networks with reference to network theory, including of nodes and edges where node represents the single user and edge represent relationship between the users [2].

The miraculous achievement of Facebook, Twitter, LinkedIn, and YouTube, social media is cover the conventional network as the primary medium for information transportation. The consolidation of user originated data and social media is the generator in the background of this innovation obstruent users generally share images, news, videos, GIFs, and opinions. As a reprecussion, considerate how users expend and inseminate the information in information dissemination process is an elementary mark approaching the construction of elective information dissemination mechanism, growing marketing and support systems on the existing media. Moreover, there are couple of major approaches of information dissemination are influence and relevance. In online media, influence as it may be capable to impinge the behaviour of remains [3]. After all, in information dissemination synopsis, influence is literally a parameter of competency of popularising information. Relevance is a section of a user and information. In other words, relevant information pleases the primary concept of information or concerns, information rehabilitation and information system of the user. $[6,13]$. The forward-looking work spotlight on coupling between the user's effectiveness and relevant information in information dissemination data, which shows how users cuddle the 
concept in concept and age. We are focusing on dissemination of information about the media, we handle 'information' and 'content' words with each other.

An essential task in the field of influence and information dissemination in online community is shortage of information at a broad suitable range. Most probably the datasets generated through online media, able to be use for new exploration have stationary topological information and do not have any main information for examine the influence and information dissemination. Moreover, social influence scrutiny needs materialistic information that shows, for example, inevitable club of users to information items. As a repercussion of aforesaid inefficiency, a compelling chunk of the models and study of influence are positioned on manufactured information, which is generally positioned on epidemiologic triad [19]. Online journal information, viral marketing campaigns, news web media, images, audios and videos distributing services and online media communities almost have furnished prosperous datasets that supported many different usable recommendations in relation to social influence and information dissemination in actual schemes.

\section{Literature Survey}

Jingxuan Li, Wei Peng, Tao Li and Tong Sun [33] Study the problem that education and industry are very interested in finding effective users and estimating their "massive social impact on a large scale" on social media. It has also been said that effective information dissemination models basically depend on the continuous - Stochastic process with Markov's property to predict the effectiveness of online media users, where node users represent in the progress of the spread. And the edges are related to users who point to some subject immediately on time. We also demonstrate that our new modelling is to be able how responsive the top influencers.

M. Cha, H. Haddadi, F. Benevenuto and K. P. Gummadi [26] expressed that by using a huge data which is gathered from micro blogging website (i.e., Twitter) and stated an exhaustive comparison of connections, retweets, and mentions which are the crucial measures of influence. According to these measures, we study the sense of user effect on logic and opportunity. We create some curious comments: (i) Highly active users who have a large number of connections are not influential. (ii) Most influential users can have consequential impact on different topics. (iii) The effect is achieved without any irregular or unknowingly, but limiting tweets in the same subject through joint efforts like. We consider that above observations furnish new visions for growing marketing and advise that particularly only indegree acknowledges very slight about a user's influence.

Sriganga B K, Ragini Krishna and Dr. Prashanth CM [1] describe that the prime concern in Social Network Analysis is to measure the centrality of nodes (entities, users) in online media. Centrality of nodes is generally used to determine the most crucial nodes and central measures of different networks have been proposed such as degree centrality that counts the numbers of neighbors a node has, closeness centrality that identify the shortest path between the node and other nodes, PageRank that is used to determine the page relevance or importance, etc. These proposed algorithms have been enforced to stationary (or static) networks. After all the actual world network is not static in general, due to their topography can diverse time to time with addition or deletion of nodes and edges. It's necessary to identify the influential node (i.e. influential user) by using network centrality for changeable networks. To attain this, we can use path-based central use in the network, which determines the centrality of the nodes based on the routes which add up to the optional nodes.

Jie Tang, Jimeng Sun, Chi Wang and Zi Yang [4] shows that nodes are influenced by others for different reasons in large social networks. For instance, co-workers have forceful influence on each other's work, although the friends have forceful influence on each other's regularly activity. How to mark social impact from discrete fields? How to compute the vitality of those social effects? How to evaluate a miniature on a real comprehensive network? To find the solution of above mentioned questions, We begin Occasional Affiliency Publicity (OAP) to minimize the social impact of problem-level on the wider network. OAP can draw the conclusions of any logic design and actual network structure to carry out the impact-level impact spread. By using influence analysis, We give details of many different essential applications on existing 
actual datasets such as: (i) What are the ideal nodes on a given issue? (ii) How to determine the social impact of neighboring nodes on a node? To extent to actual broad structures, OAP is constructed with useful distributed learning algorithms which have been enforced and certified under MAP-Reduce scheme. We also show the basic aspect of distributed learning algorithms for MAP-Reduce. Finally, we mark the ability and adaptability of OAP on actual huge datasets.

Haewoon Kwak, Changhun Lee, Hosung Park and Sue Moon [23] begin with the network analysis and study the distribution of followers and follow-up, relations between followers and tweets, different degrees. After this we present many followers, many users and many Page-Rank and quantitative comparison between them. Ranks by tweets push more than millions of followers who are more than millions of followers. Through the analysis of our topic, we show that trending topics are classified in different categories, for how long they and how many users participate. In the end, we study the information dissemination by retweet. We build the tree rings and test their spatial and temporal characteristics.

Arlei Silva, Herico Valiati, Sara Guimaraes and Wagner Meira Jr. [30] expressed that intimate demeanor data can furnish knowledge about communications in a media and also defines how We affect network discovery issues, during which time the relationships affecting user behavior to determine. Several strategies are recommended for impact network search, and using a Twitter Followers Network and User Activity Data on a case study, the application of such strategies is discussed, which is a popular micro-blogging and social networking site. We believe that follower-compliant contacts define the connection to the potential impact between the user and the posting work of tweets, URLs or hash-tags shows a personal behavior on Twitter. Results show that tweets can be used effectively in search of effect relations, hash-tags and URLs, such tasks do not get good performance. Apart from this, the strategies, which at the time considered when a person's behavior is made better than those who do not combine with the popularity of behavior, the information aforesaid, and better results can be obtained.

Arlei Silva, Sara Guimaraes, Wagner Meira and Mohammed Zaki [22] study the issue of determining effective users and admissible information in information dissemination data. We recommend Profile-Rank, a unique information dissemination model based on arbitrary affinity to a user's information content. Profile-Rank is a Page-Rank driven model that interpret the theory that admisible information is constructed and promoted by influential users and effective users have made residual information. An important charaterstic of Profile-Rank is that it can be customized to purnish actualize endorsements. Empirical outcomes show that Profile-Rank creates precise recommendations, performs better baseline techniques. We also show admisible information and influential users using Profile-Rank. Our analysis shows that ProfileRank scores are more correlated with information dissemination than network structure. We also begin to calculate that our new modeling with Page-Rank is more efficient.

\section{Conclusion}

In this paper, we study how personal behavioral data can be implemented in the recognization of impact relations in online media. This paper conducts various research papers which propose many different methods which are the highly useful in the area of information dissemination data for imminent exploration. Determining large datasets by efficient user are the interesting assignments in the area of information dissemination data. Profile-Rank is an offhand running information dissemination model which uses the information dissemination data to calculate user influence and admissible information. Profile-Rank scores are related to the spread of more information dissemination than network-structure. We also explained that new approch from Page-Rank is more efficient.

\section{References}

[1] Sriganga B K, Ragini Krishna, Dr. Prashanth CM, et al, / (IJCSIT) International Journal of Computer Science and Information Technologies, Vol. 5(3), 2014, 4047-4053

[2] Pinheiro, Carlos A.R. Social Analysis in Telecommunication. John Wiley \& Sons. p 4. ISBN 978-1118-01094-5., 2011

[3] N. Friedkin, A structural theory of social influence, volume 13, Cambridge University Press, 2006.

[4] J.Tang, J. Sun, C. Wang, and Z. Yang, Social influence analysis in large-scale networks, In KDD, 2009. 
[5] R. Baeza-Yates, B. Ribeiro-Neto, et. al. Modern information retrieval, volume 82. Addisom-Wesley New York, 1999.

[6] F. Alkernade and C. Castaldi, "Strategies for the diffusion of innovations on social networks", Comput. Economics, vol. 25, no, 1-2, pp. 3-23, 2005.

[7] M. Gomez Rodrigues, J. Leskovec, and A. Krause. Inferring networks of diffusion and influence. In SIGKDD, 2010.

[8] H. Tong, S. Papadimitriou, P.S. Yu, and C. Faloutsos. Proximity tracking on time-evolving bipartite graphs. In SDM, 2008.

[9] A. Goyal, F. Bonchi, and L.V. Lakshmanan. Learning influence probabilities in social network. In WSDM, 2010.

[10] D. Gruhl, R. Guha, D. Liben-Nowell, and A. Tomkins. Information diffusion through blogspace. In WWW, 2004.

[11] J. Hannon, M. Bennett, and B. Smyth. Recommending twitter users to follow using content and collaborative filtering approaches. In RecSys, 2010.

[12] F. Ricci, L. Rokach, and B. Shapira. Introduction to recommender systems handbook, 2011.

[13] H. Tong, C. Faloursos, and J. Y. Pan. Fast random walk with restart and its applications. In ICDM, 2006.

[14] J. Weng, E. P. Lim, J. Jiang, and Q. He. Twitterrank: finding topic-sensitive influential twitterers. In WSDM, 2010.

[15] A. Anagnostopoulos, R. Kumar and M. Mahdian. Influence and correlation in social networks. In KDD, 2008.

[16] D. Kempe, J. Kleinberg, and E. Tardos. Maximizing the spread of influence through a social network. In PKDD, 2011.

[17] J. Yang and J. Leskovec. Patterns of temporal variation in online media. In WSDM, 2011.

[18] M. R. Subramani and B. Rajagopalan. Knowledge-sharing and influence and passivity in social media. In PKDD, 2011.

[19] B. Taskar, M. Fai Wong, P. Abbeel and D. Koller. Link prediction in relational data. In NIPS, 2004.

[20] D. Watts and P. Dodds. Influentials, networks and public opinion formation. Journal of consumer Research, 2007.

[21] D. M. Romero, W. Galuba, S. Asur and B. A. Huberman. Influence and passivity in social media. In PKDD, 2011.

[22] Arlei Silva, Sara Guimaraes, Wagner Meira Jr., Mohammed Zaki, "ProfileRank: finding relevant content and influential users based on information diffusion, 2013.

[23] H. Kwak, C. Lee, H. Park, and S. Moon. What is twitter, a social network or a news media? In WWW '10: Proceeding of the $19^{\text {th }}$ International conference on WWW, pages 591-600, 2010.

[24] M. J. Pazzani and D. Billsus. Content-based recommendation systems. In The Adaptive Web, pages 325-341. Springer Verlag, 2007.

[25] N. E. Friedkin, A structural theory of social influence. Cambridge University Press, 1998.

[26] M. Cha, H. Haddadi, F. Benevenuto, and K. P. Gummadi. Measuring User Influence in Twitter: The Million Follower Fallancy. In ICWSM, 2010.

[27] E. Bakshy, B. Karrer, and L. A. Adamic, "Social influence and the diffusion of user-created content", in proceedings of the $10^{\text {th }}$ ACM conference on Electronic commerce. Acm, 2009, pp. 325334.

[28] M. Gomez Rodriguez, J. Leskovec. And A. Krause, "Inferring networks of diffusion and influence", in proceedings of $6^{\text {th }}$ ACM SIGKDD international conference on Knowledge discovery and data mining. ACM, 2010, pp. 1019-1028.

[29] FP. Bonchi, "Influence propagation in social networks: A data mining perspective". IEEE Intelligent Informatics Bulletin, vol. 12, no. 1, pp. 8-16, 2011.

[30] A. Silva, H. Valiati, S. Guimaraes, and W. Meira Jr. From individual behavior to influence networks: A case study on twitter. In Webmedia, 2011.

[31] E. Bakshy, I. Rosenn, C. Marlow, and L. Adamic, "The role of Social networks in information diffusion", in Proc. Of the $21^{\text {st }}$ international conference on WWW. ACM, 2012, pp, 519-528.

[32] S. Aral, L. Muchnik, and A. Sundararajan. Distinguishing inflence-based contagion from homophilydriven diffusion in dynamic networks. PNAS, 2009. 
[33] M. De Choudhury, S. Counts, and M. Czerwinski. Identifying relevant social media content: leveraging information diversity and user cognition. In HT, 2011.

[34] J. Wortman, "Viral marketing and the diffusion of trends on social networks", University of Pennsylvania, Tech. Rep. Technical Report. MS-CIS-08-19, May 2008.

[35] Jingxuan Li, Wei Peng, Tao Li, Tong Sun. SocialNetwork User Influence Dynamics Prediction. Pp 310-322, Springer - Verlag Berlin Heidelberg, 2013.

[36] Fredrik Erlandsson, Piotr Broadka, Anton Borg, Henric Johnson. Finding Influential Users in social media using Association rule learning. Entropy 2016, 18, 164; doi: 103390/e18050164. 